\title{
Complexity and Accountability: The Witches' Brew of Psychiatric Genetics
}

\begin{abstract}
This paper examines the role of complexity in descriptions of the aetiology of common psychiatric disorders. While scientists attest to the discovery of an underlying reality of complex inheritance - the so called 'witches' brew' of genetic and non-genetic factors - we argue that 'complexity' also performs rhetorical work. In our analysis of scientific review articles (1999-2008), we find a relatively stable genre of accountability in which descriptions of complexity appear to neutralise past failures by incorporating different and sometimes competing methodological perspectives. We identify two temporal strategies: retrospective accounting, which reconstructs a history of psychiatric genetics that deals with the recent failures, citing earlier twin studies as proof of the heritability of common psychiatric disorders; and prospective accounting, which engages in the careful reconstruction of expectations by balancing methodological limitations with moderated optimism. Together, these strategies produce a simple-to-complex narrative which belies the ambivalent nature of complexity. We show that the rhetorical construction of complexity in scientific review articles is oriented to bridging disciplinary boundaries, marshalling new resources and reconstructing expectations that justify delays in gene discovery and risk prediction.
\end{abstract}

Keywords: complexity, psychiatric genetics, accountability, rhetoric 
After the disappointing failure of the 'gene for' paradigm, it appears that scientists working within the field of psychiatric genetics no longer adopt a deterministic view of common psychiatric disorders. Conditions such as schizophrenia and bipolar disorder are now considered complex traits. Such models of genetic complexity have gained significant ground, leaving few psychiatric traits or conditions without a putative biological explanation.

In the context of the controversies that have dogged this field, we suggest that this turn to complexity accomplishes more than merely provide a description of the natural world, it also performs rhetorical work. Biomedical scientists appear to be engaging in the management of intellectual responsibility, what we call 'professional accountability'. In this paper, we show the ways in which narratives of psychiatric genetics employ a variety of invocations of complexity to present psychiatric genetic research as cautious, flexible and responsible science. In the narrative of past failure and future progress, notions of complexity provide theoretical coherence and respectability to an otherwise ambivalent relationship between genetic and nongenetic factors.

There is a literature on genetics, rooted in the social sciences, which is critical of the claims of the new genetics (Yoxen, 1982; Lippman, 1991; Cranor, 1994; Katz Rothman, 1995). This paper is not an explicit contribution to this literature; it is not a critique of the reductionism of psychiatric genetics. Rather, we adopt a symmetrical stance towards hypotheses of genetic causation by focussing on the patterns of accounting within a particular genre of the literature of psychiatric genetics that underwent a growth in the late 1990s and early 2000s. These articles, locating psychiatric genetics in an optimistic history, come after the failures of the gene identification programmes of the 1990s, at a time when the Human Genome Project (HGP) promised new informational and technological possibilities.

The most straightforward understanding of the relationship between simplicity and complexity is that of oppositional poles on a continuum; that complexity is merely a quantitative increase in 'complicatedness'. However, the turn to complexity is sometimes enthusiastically presented in terms of a 'paradigm-shift': a Kuhnian revolution in which linear and reductionist tools and methods of are replaced by nonlinear and dynamic counterparts that are capable of grasping complex objects. In contrast with these visions of an a priori objective reality, this paper does not treat complexity as a thing in itself, but as a theme in rhetorical/descriptive practices of scientific accounting.

The turn to complexity provides new forms of justification. We demonstrate that invocations of complexity in psychiatric genetics do not replace the simple, reductionist genetic hypothesis, but provide the means to rescue research ventures into genetic causation from the failures of the past. The development of a vision of post-genomic complexity is accomplished through innovative strategies and techniques of persuasion and fact construction. Psychiatric genetics provides a case study of the defence of a controversial science: in this case, narratives drawing on 
complexity account for past failures and neutralize criticisms of genetic determinism by incorporating both the unknown and 'non-genetic' factors within their theoretical models.

\section{Controversy and Hereditarian Ideas in Psychiatry}

In this section, we consider some of the controversies that have shaped psychiatric genetics, most of which stem from the central claim that common psychiatric disorders have a significant heritable component and explained by underlying genetic factors. The history of controversy in psychiatric genetics has many starting points. The most relevant for our present inquiry are those emerging from hereditarian ideas that informed early eugenic programmes during the midtwentieth century (Kevles, 1995; Kerr \& Shakespeare, 2002). More recently, we have seen the reintroduction of biological determinism into discussions of human behaviour beginning with Wilson (1975) and extending into popular thought via Ridley (1994, 1996), and Wright (1996). The extension of 'sociobiology' and 'evolutionary psychology' has been also challenged from within the scientific community following Rose, Lewontin and Kamin's (1984) high profile critique Not in Our Genes.

In psychiatric genetics itself, twin studies are routinely enrolled by the field as providing the strongest evidence that genetic factors play a major role in the causation of psychiatric disorders. However, controversy has surrounded their role in supporting the genetic bases of psychiatric conditions such as schizophrenia and bipolar disorder. Criticisms of twin studies usually focus on aspects of methodology and statistical calculation (Kamin, 1974; Wahlsten, 1979; Plomin, 1990; Billings et al. 1992; Joseph, 2001, 2003, 2006) although the fraudulent research of Cyril Burt adds further scandal to the mix (Rose et al., 1984). However, Hedgecoe (2001) has remarked that critics often fail to take into account the 'time lag' between old and new generation twin studies. Critics often focus on older twin studies (pre-1994) to ground claims that twin studies are methodologically flawed, while new generation twin studies claim to be 'constantly evolving and improving' (Winerman, 2004). They also claim to be more sensitive to complex traits by incorporating 'multivariate' and 'covariate' analysis (Boomsma et al., 2002).

This paper has little concern with whether or not twin studies are actually or intrinsically flawed. Rather, our focus is the way in which the 'simplicity' of twin studies is recruited by the field in ways that seem to both endorse complexity and minimize controversy. A more recent controversy is the failure of genetic linkage studies to find 'genes for' psychiatric disorders. We concentrate on this period because it is in part these failures which are being accounted for in the articles we examine.

\section{Linkage Studies}

Technological developments in the 1970s and 1980s provided the foundations for great optimism in gene identification research programmes. The development of recombinant DNA technologies and advances in chromosomal mapping allowed researchers to develop linkage maps to identify the relative positions of mutations. A 
precursor to the HGP, linkage maps led to 'highly competitive races' to identify genetic mutations 'for' diseases (Cook-Deegan, 1994).

Amidst the high-profile successes of this period - high points include the identification of the 'genes for' cystic fibrosis in 1989 and Huntington's disease in 1993 - psychiatric genetics was inspired to apply the same techniques to locate genes for common psychiatric disorders. A raft of highly visible papers reported successes in using linkage studies to identify, amongst others, 'genes for' schizophrenia (Sherrington et al., 1988), bipolar disorder (Baron et al., 1987; Egeland et al., 1987), and alcoholism (Blum et al., 1990). But success soon turned to embarrassment when other teams failed to replicate findings for schizophrenia (Kennedy et al., 1988), manic depression (Detera-Wadleigh et al., 1987; Hodgkinson et al., 1987), and alcoholism (Gelernter et al., 1991, 1993). Authors of two of the 'successful' linkage studies that had claimed to find a 'gene for' manic depression published retractions in Nature (Kelsoe et al., 1989; Baron et al., 1993). As one commentator has noted, events of this kind are 'unusual and embarrassing ... among scientists' (Berkowitz, 1996: 43).

Our post-mortem of one linkage study highlights the difficulties in claiming simple genetic causation. Egeland and colleagues chose a relatively isolated and homogenous sample for their linkage study of manic depression: an Amish community in Pennsylvania. In 1987, they published their findings in Nature, confidently asserting that 'a dominant gene conferring a strong predisposition to manic depressive disease' (Egeland, et al. 1987: 783) had been found on chromosome 11. However, in the same issue, two related studies found no linkage between the genetic markers identified and manic depression in three Icelandic families (Hodgkinson et al., 1987) and three North American families (DeteraWadleigh et al., 1987). While Nature was careful to report these discrepancies, the Egeland study clearly occupied the spotlight, with the editor asserting that 'the use of DNA markers has shown that manic-depressive illness can be caused by a single gene' (Robertson, 1987: 755).

In a later issue of the same year, Baron et al. (1987) reported close linkage between manic depression and a region of DNA on the $X$ chromosome. The researchers concluded: '[t]hese results provide confirmation that a major psychiatric disorder can be caused by a single genetic defect' (Baron et al., 1987: 289). Despite the difficulties of replication and of genetic heterogeneity, there was a strong trend within the field to support a simple model of genetic causation for manic depression. However, by 1989 readers of Nature would have detected a tidal change in the waves of retraction and reevaluation of the single gene hypothesis. For instance, Egeland's group published a reevaluation of their own findings (Kelsoe et al., 1989) based on a change in diagnosis for two family members as well as new data from additional families. Two members of the Amish pedigree received a diagnosis of manic depression in the absence of the putative markers and an examination of an additional branch of the original pedigree found evidence against linkage. This reversal suggests that a theoretical model of genetic heterogeneity cannot, in itself, explain away or defend the single gene hypothesis. 
In acknowledging this 'false start on manic depression', the editor of Nature conceded that the hypothesis that 'a gene on chromosome 11 could predispose to manic depression was calculated on a delicate balance of uncertainties' (Robertson, 1989: 222). Such failures to replicate, it was argued, 'highlight many of the problems that can be anticipated in genetic linkage studies of common and complex neuropsychiatric disorders' (Kelsoe et al., 1989: 242).

In light of such problems, the field of psychiatric genetics began to adopt a more complex model in which psychiatric disorders are understood to be the product of several genes, which may be interacting non-additively, each with only a small effect, with different variations in each family (Gershon \& Cloninger, 1994). Technological developments in the late 1990s and 2000s, such as high-density SNP (Single Nucleotide Polymorphisms) maps and high-throughput genotyping, derived from the HGP, provided the foundations for great optimism in the potential to genetically dissect complex disease. The articles analysed in this paper are drawn from the bloom of review articles that provide accounts of this optimistic turn. However, the advent of complexity within this field is not a 'paradigm shift'. The shift from 'genes for' to 'susceptibility genes' involves a scaling up of gene identification programmes without necessarily incorporating notions of nonlinear complexity.

\section{Rhetoric in Scientific Writing}

The term 'rhetoric' is often narrowly understood as the techniques of argumentation and persuasive communication (Cockcroft \& Cockcroft, 1992). Drawing from the work of Michael Billig (1987) and Jonathan Potter (1996), we define rhetoric more broadly as the everyday organization of descriptions and explanations, and their relation to different and competing versions of the truth. This relational dimension of rhetoric is particularly effective for the analysis of controversies: in the way that constructions of fact and description subtly or explicitly counter alternative descriptions (Dillon, 1991). Scientific controversies can be analysed in similar ways to evaluate the techniques of rendering factual accounts robust and persuasive. Descriptions of fact are reworked and reframed to undermine competing accounts, and protected or insulated from counter claims. For the purposes of our own analysis, we suggest that the activity of formulating scientific accounts as descriptions of reality take on both an action and an epistemological orientation (Potter, 1996).

An example of these orientations at work can be found in Gilbert and Mulkay's (1984) classic analysis of scientists' discourse. They show that scientists draw upon different 'interpretive repertoires' to describe scientific controversies and discoveries in the context of research interviews. Interpretive repertoires specify distinctive vocabularies and grammatical styles of description and explanation. They noted that scientists characteristically use an 'empiricist repertoire' to describe their work as factual and objective. The empiricist repertoire treats data as primary and deflects attention away from scientists' own speculations and beliefs. When scientists referred to themselves informally, agency was often described as constrained by rules or by the actual phenomenon under investigation. A recurring 
feature of the empiricist discourse is the way that descriptions reify their objects, making them seem solid and real. Latour and Woolgar (1986) have documented the resources that scientists use to work up the factuality of descriptions. For instance, what they call the 'hierarchy of modalization' is integral to processes of fact construction. Low-modality ('X is ...', 'we know X ...') treat descriptions as factual and unequivocal, while high-modality descriptions (' $X$ is possible', ' $X$ is likely') are treated as provisional and uncertain. Notions of certainty and contingency are not simply competing strategies that either bolster or undermine scientific authority; they can also be combined to produce cautious, and therefore responsible, displays of professional authority.

While research interviews are interesting in their own right for showing how scientists' claims are subtly undermined by the contingency of their own verbal statements, written accounts of science have broader rhetorical reach. We believe that journal articles provide an important site for evaluating the rhetorical dimension of language, the analysis of which helps us to understand the survival of psychiatric genetics as a particular discursive style. For this reason, we focus our rhetorical/discursive approach on the analysis of review articles.

The review article has become an important form of writing in the sciences, especially where there have been steady increases in published information and rapid developments in research (Virgo, 1971; Bazerman, 1988). Analyzing these articles is constructive for two reasons. First, they can be considered the 'official' descriptions of scientific activity; their credibility as exemplary statements of the field is sanctioned by processes of peer review. Second, these articles reveal a relatively stable genre of professional accounting. Greg M yers (1991) has argued that writers of review articles give the literature of a field a narrative form, inviting readers to extend and continue the story. As the story of the field is developed, key events and controversies are routinely cited (or omitted) in ways that provide coherence and stability. Christine Sinding (1996) has also noted that review articles can be treated as a literary genre that provide opportunities for constructing and reconstructing knowledge claims. Review articles are important not only because they provide information but because they engage in multiple activities such as popularization (Hilgartner, 1990), fact construction (Myers, 1992), and narrative reconstruction (Hedgecoe, 2001). Indeed, it is the action orientation of speculative and programmatic claims that seek to bridge disciplinary boundaries, gather allies and reshape the past. Our work extends on this tradition of textual analysis, taking the review article as a clearly emerging genre of scientific accountability.

\section{Methods}

This paper emerges from our ethnographic involvement with the field of psychiatric genetics, in particular with a major UK research centre. Reading the literature of psychiatric genetics, we identified a particular genre of review writing. These articles are neither research papers nor reviews of research, but locate the field in a narrative of development. The period of time considered in this paper, 1999-2008 (inclusive), is a product of the flourishing of this genre during the 1990s as the HGP neared completion. 
The first stage of our sampling strategy was a combined search of PubM ed and ISI Web of Knowledge using the simple search string 'psychiatric genetics'. As noted, we limited our search to articles published between 1999 and 2008. This generated 906 journal articles. The titles, and where necessary the abstracts, of these articles were examined to identify a particular type of review article. Such articles include a clear temporal narrative, accounting for the past and speculating on the future of the field. A common feature is the building of a narrative of progress, often explicitly signalled by the temporal framing of their titles - e.g. 'The future of psychiatric genetics', 'Back to the future', and 'Future strategies of psychiatric genetics'. The peculiarity of this genre of scientific writing is highlighted by the variety of ways in which journals accommodated these articles into their existing categories, being described as 'reviews', 'updates' or 'editorials'. It was notable that the specialist journal, Psychiatric Genetics, carried no such review articles.

This process produced a sample of 35 articles. A process of systematic reading and note-taking identified common themes and rhetorical devices. These were coded for further analysis. During this analysis, the invocation of 'complexity' was identified as a key feature of these articles. Therefore, the uses of complexity emerged as the analytic focus around which more detailed readings were organized. The journals from which these articles were drawn (See Table 1) cover diverse disciplines, indicating the transdisciplinary origins of psychiatric genetics, the different contexts in which the articles are located, and the range of audiences.

Table 1. Journals from which articles were drawn

\begin{tabular}{|l|l|}
\hline \multicolumn{1}{|c|}{ Journal } & \multicolumn{1}{|c|}{ Field } \\
\hline Nature & General life science \\
The Lancet & General medicine \\
Trends in Genetics & General genetics \\
Genetics in Medicine & Medical genetics \\
American Journal of Medical Genetics & Medical genetics \\
European Neuropsychopharmacology & Biological aspects of psychiatry \\
Molecular Psychiatry & Biological aspects of psychiatry \\
International Review of Psychiatry & Psychiatry \\
Annals of Clinical Psychiatry & Psychiatry \\
American Journal of Psychiatry & Psychiatry \\
British Journal of Psychiatry & Psychiatry \\
& \\
\hline
\end{tabular}

Following this preliminary analysis, 10 articles were selected for detailed rhetorical/discourse analysis. Purposeful sampling was used to include articles published across the time period and a spread of journals representing a range of specialties, audiences, and locations (UK, USA and Canada).

In the following section, we describe the main rhetorical themes in these articles, and present key extracts to demonstrate the devices and techniques used to 
construct the narrative of the field. Where extracts are reproduced, the use of italics adds emphasis for illustrative effect.

\section{Accounting with Complexity}

A common element of the narrative of psychiatric genetics is the emergence of complexity in accounting for past failures, warranting delays in gene discovery, and justifying new methods. We identify two temporal strategies through which a narrative of psychiatric genetics is organized:

- Retrospective accounting: in which accounts are oriented to manage the failures of genetic linkage studies. This strategy involves the routine citation of twin studies as evidence to defend the genetic hypothesis;

- Prospective accounting: in which accounts are oriented to the careful construction of the future, invoking the technical difficulties of complexity to moderate optimistic expectations.

All the articles examined contain these temporal strategies that navigate a narrative of problems, progress and potential. In this section, we show that descriptions of complexity are composed of competing assertions about the progress of psychiatric genetics. The emerging view of complexity is one that attributes increasing significance to polygenic, multifactorial causation, distancing the field from a simple Mendelian model of inheritance. Yet, as we shall see, this view still retains an interest in searches for single genes associated with psychiatric disorders with medium-to-large effects.

One way to accommodate the 'mixed findings' of previous studies into a coherent model of complexity is to construct these descriptions as 'factual' accounts. We found that the authors within our sample used a number of rhetorical techniques to direct attention away from speculations within the field and towards the 'objective' mechanisms of disease. An early example of such processes can be found in the high impact journal M olecular Psychiatry:

\footnotetext{
However, common illnesses pose much greater challenges for geneticists because, in the majority of cases, they result from the combined action of a number of different genes (each of which may result in only a modest increase or decrease in liability) as well as environmental influences; a witches' brew termed polygenic, multifactorial causation. Further complexities include the possibility of non-additive genetic effects, including gene-gene interactions (epistasis), and also potential gene-environment interactions . (Owen et al., 2000)
}

We found that authors who presented the most circumspect accounts of progress were often collaborators in the new generation of genome wide association (GWA) studies. Working up complexity into a 'fact' provides justification for the collection of large samples to identify small-effect genes for common psychiatric disorders. Making this case requires distancing common illnesses from the study of large-effect genes. This is accomplished by contrasting rare, monogenic disorders ('However ...') from 'common illnesses', which 'pose much greater challenges for geneticists'. The transformation of speculation into factual descriptions is accomplished by casting complexity of common illnesses as the primary 'complicating' agent (e.g. 'they result from the combined action'). Complexity is described as the effects of multiple gene 
interactions and 'potential gene-environment interactions'. While this offers a more definite description of complexity than some accounts, there is a tension between uncertainty and object-reality. Various markers of modalization ('may result in', 'possibility of', and 'potential') suggest that the nature of disease action is provisional and uncertain, rather than emphasizing the authority of scientists or their claims that genetic causation is complex. Such factual descriptions of complexity transfer speculation and uncertainty from the field to the disorders. Complexity becomes a constitutive part of the phenomena being studied. These processes of reification and ambivalence are recurrent features of descriptions in which new methods are proposed. Below is another example, published in the journal Human Molecular Genetics during the same year:

The transmission patterns of psychiatric disorders are undeniably complex. It is likely that a variety of genetic as well as environmental pathways can increase one's susceptibility to a given psychiatric disorder. This is the concept of equifinality, where different initial conditions can lead to the same endpoint [...] it is anticipated that to increase risk for many complex disorders, multiple deleterious genetic variants are required in combination. This is called multiplicative, epistatic, oligo- or multigenic inheritance. (Stoltenberg \& Burmeister, 2000: 927)

Addressing an audience well versed in methodological issues but not specialist in psychiatric disorders, this description forms part of the growing realization that many susceptibility alleles will be common variants rather than rare mutations. Again, the existence of complexity appears to justify new methodological approaches of psychiatric genetic studies. Claiming that psychiatric disorders 'are undeniably complex' persuasively orients to an apparent consensus in the field. Markers of modalization ('It is likely that ... can increase') mitigate commitment to the provisional assertion of gene-environment interaction as an explanation for increased susceptibility. This is formalized by the propositional statement: 'This is the concept of equifinality'. Impersonal constructions ('it is anticipated that') produce a similar effect. They allow authors to distance themselves from conjecture when describing epistatic inheritance. Any sense of conjecture or interpretation is glossed by the propositional statement that follows ('This is called multiplicative ... inheritance'). These formal descriptions of complexity succeed in transforming 'disease complication' into a reified phenomenon.

All the articles in our sample employed this multifactorial model of complexity to make claims about promising new strategies and methods. This approach has the benefit of confirming the genetic hypothesis while at the same time eschewing reductionism by enrolling non-genetic factors. However, such descriptions of complexity were frequently accompanied by ambivalence and moderation, which points to a curious relationship between simple Mendelian and multifactorial models. If psychiatric disorders are 'undeniably' complex, why do authors need to routinely account for non-M endelianism?

To answer this question, we turn to two articles that illustrate competing accounts of methodological directions within psychiatric genetics. The first article, in the high impact journal Trends in Genetics, presents a methodological argument to a broad audience of geneticists. Evans et al. (2001) describe how linkage studies can 
overcome phenotypic heterogeneity (poorly defined diagnostic boundaries) by carrying out analyses under several diagnostic definitions. In the case of schizophrenia and bipolar disorder, diagnostic complexity may account for the clinical similarity of these disorders. Linkage studies of large family sets have produced 'mixed results', confirming that psychiatric disorders 'cannot entirely be accounted for by one or a few major loci' (Evans et al., 2001: 36). However, rather than dismissing linkage approaches, Evans et al. argue for combining linkage analysis with models of 'complex inheritance':

\begin{abstract}
Although linkage analysis has failed to detect loci in a number of study sets, it is striking that several genome-wide scans, in both single extended pedigrees and large sets of families and sibling pairs, have identified loci showing highly significant linkage. It seems probable, therefore, that susceptibility to psychiatric illness reflects a mixture of the two genetic models. (Evans et al., 2001: 36)
\end{abstract}

Notions of complexity are usually invoked to distance the new generation of research from Mendelianism, but this passage describes how genome-wide scans, designed to detect genes of small effect, can be used to identify genes of large effect. The methods used to explore complex genetics also detect Mendelian-like ('highly significant linkage') genes. This mixing of simple and complex models seems at odds with claims that complex genetics incorporates environmental factors, claims which neutralize criticisms that genetic psychiatry is reductionist (cf. Rose, 1995; 1998). In fact, this is precisely the strategy that Evans et al. employ towards the end of their article:

Do genetics and genomics hold all the answers? Some commentators challenge the
whole reductionist approach of genetics applied to psychiatry, dismissing it as a
distraction from the importance of social and cultural influences. In truth, studies of
genetic modulation and/or genetic variation are perfectly well suited to explore
environmental influences, and to move beyond mere description towards a mechanistic
and mathematical understanding of biological connectivity and emergent properties.
(Evans et al., 2001: 39)

The accusation that psychiatric genetics is a 'distraction' from research into sociocultural factors is countered by attributing agency to 'studies of genetic modulation and/or genetic variation'. It is not the scientists who have to account for 'social and cultural influences', the studies themselves will 'explore environmental influences'. This move from 'mere description towards a mechanistic and mathematical understanding' may not satisfy the critics if 'social and cultural influences' are reduced to an algorithm.

While Evans et al. (2001) are excited about the prospects of combining linkage and association studies, Kendler (2006) offers a more circumspect account of progress. Writing in the American Journal of Psychiatry, the most widely cited psychiatric journal, Kendler is sceptical of the nosological validity of family studies, suggesting that family aggregation has a tendency to produce false positives because it assumes that affected members have the same single dominant mutation. He suggests that rather than studying one disorder at a time, it may be more useful for nosologists to see if family aggregation occurs across or within diagnostic boundaries. However, 
even if such studies confirmed that two disorders shared genetic risk factors, what explanatory value does this provide? Kendler is sceptical whether molecular genetics will provide the same clarity to classifications in psychiatry as it has for other fields of medicine. He asks whether psychiatry is at risk of adopting a 'gene-centred' approach that inflates the diagnostic value of individual genes of 'modest effect sizes'.

Kendler's critiques are unusual for an established scientist who speaks from within psychiatric genetics. He observes that the optimism of the field is closely linked to an 'essentialist model' that assumes an underlying genetic basis for psychiatric categories. Such models are attractive and simple to teach: 'They fit well into the traditional medical model, thereby supporting the status of psychiatry as a medical discipline ... they provide support for an organic disease model where psychiatric disorders are understood as resulting from pathological processes in the brain' (Kendler, 2006: 1141). Outlining the progress of the field, Kendler questions whether the way forward is to identify genes and then 'work our way back' to ground diagnostic categories on the foundation of a gene:

\footnotetext{
By 2004, it had become clear to everyone in the field that no 'M endelian-like' genes for psychiatric disorders were likely to be found. Nonetheless, there is continued hope that advances in psychiatric genetics and particularly the identification of individual susceptibility genes will alter, in fundamental ways, our approach to psychiatric diagnosis. If we are able to find a 'gene for' a particular psychiatric disorder, then we can work our way back up and - as predicted by the EGM [Essentialist Gene Model] ground our diagnostic category on the firm foundation of a gene. (Kendler, 2006: 1142)
}

This extract employs a number of techniques that disrupt accounts of progress. Descriptions of the recent past ('By 2004...') build a factual account via consensus: 'it had become clear to everyone in the field'. The failure to find ' $M$ endelian-like genes' is presented as historical fact. Furthermore, the avoidance of impersonal constructions does not impute agency to theoretical models or empirical evidence, but to the responsibility of individuals who seek to ground diagnostic categories on a genetic foundation ('our approach', 'we are able to', 'we can work', 'our diagnostic category'). Another strategy is to establish a correspondence between 'M endelianlike genes' and 'the identification of individual susceptibility genes'. Kendler argues that while 'susceptibility genes' draw on notions of complexity to acknowledge the multifactorial dimension of psychiatric disorders, the lingering hope of seeking 'individual' genes is consistent with the essentialist model.

Kendler's account of the complexity of psychiatric disorders exercises far greater caution than typical descriptions of complexity. 'Gene sharing', where the same gene can contribute to the production of dramatically different phenotypic effects, frustrates attempts to isolate genetic causation. The functional boundaries of the gene are 'blurred' by processes that alter the structure of expressed proteins. Further, the physical boundaries of the gene are blurred by the role of regulatory and control regions (i.e. 'promotors') which play an important, yet under described, role in gene expression. Rather than discrete entities, genes are seen as being 'dynamic parts of biological systems of immense complexity' (Kendler, 2006: 1144). In Kendler's view, it makes little sense to search for the specific genes involved in the 
aetiology of psychiatric disorders because it is unlikely that these can offer the basis upon which to build a discrete categorical model of psychiatric diagnosis.

As we have seen, descriptions of complexity appear within key journals, providing not only an explanation for the failure of Mendelian inheritance, but also a justification for employing new generation GWA studies. The solidification of accounts of complexity into a coherent theoretical account cements methodological support for studies designed to find multiple genes of small effect. Despite the stability offered by such theoretical descriptions, there is considerable ambivalence about the nature of complexity in psychiatric disorders. This ambivalence permits interpretive flexibility. The continuing focus on finding single genes could be thought of as a product of lingering methods that are designed to find single genes, rather than a lingering commitment to Mendelian ways of thinking. No matter the degree to which a multifactorial model proposes a complex relationship between genetic and non-genetic factors, the dominant essentialist view always allows single genes to play some role in the complex aetiology of psychiatric disorders.

\section{Retrospective Accounting}

In this section, we show how authors routinely invoke historical accounts of genetic research to accomplish a number of rhetorical strategies. These strategies can be understood if we consider the implicit charge that the field of psychiatric genetics has failed to demonstrate the genetic basis of psychiatric disorders. Retrospective accounting is a strategy for neutralizing such charges by defending and/or justifying the genetic hypothesis.

A common pattern of accounting, appearing in a wide range of journals from general medicine to specialist genetics, from clinical to molecular psychiatry, involved authors revisiting the findings of twin and adoption studies. If contemporary notions of complexity are characterized by the multiplication of ambiguous and confounding factors, the same cannot be said about the early studies of heritability, which are comparably simple and well replicated. The following selection briefly illustrates this pattern of accounting:

It is now clearly established, on the basis of results from family, twin and adoption studies, that genetic factors play a major role in the etiology of schizophrenia. (O’Donovan \& Owen, 1999: 587)

\footnotetext{
Nevertheless, in contrast to some other complex disorder no susceptibility loci for psychiatric disorders have been unambiguously identified. This is especially disappointing given the overwhelming epidemiological evidence that susceptibility to psychiatric disorders has a substantial genetic component. (Stoltenberg \& Burmeister, 2000: 927)

In relation to substance use, twin studies provide unambiguous evidence that genes play an important role in the development of alcohol dependence. (Dick, Rose \& Kaprio, 2006: 224)
}

A characteristic of the above examples is their high modalization and extreme formulation to construct a factual account that psychiatric disorders have a genetic component. These claims of unequivocal certainty are curious: none of the articles 
mention the methodological flaws and biases of twin and adoption studies (discussed earlier in this paper), which is interesting because some of these criticisms have been raised from within the field (Kendler \& Diehl, 1993). Hedgecoe (2001) has observed that in the genetics of schizophrenia, authors provide selective accounts of twin and adoption studies which elide such criticisms and bring closure to an area of controversy.

The invocation of twin and adoption studies also provides a continuous history, connecting contemporary research with the successes of the past, by which present concerns about the ambiguity of genetic complexity can be moderated. We identify two kinds of formulation in which simple history and complex present are contrasted to achieve persuasive results. The two formulations differ in the strength of their assertion of the genetic hypothesis. The 'moderate' formulation consists of the following sequence of claims:

1. Identifying genes for psychiatric disorders is difficult.

2. This is because psychiatric disorders are complex.

3. However, history shows that psychiatric disorders are heritable.

The sequence begins with the assertion/concession that gene-identification for psychiatric disorders is difficult. This claim warrants an account ('This is because...'), which is supplied as a contrast in the second part of the sequence: psychiatric disorders are characterized by complex causation. The second part raises ambiguity about the specific role of genetic factors, whether they are single genes of dominant effect or multiple genes of small effect. Environmental influences are also included as playing a 'complicating' role to account for these difficulties. The final part of the sequence introduces another contrastive statement that reconfirms the genetic hypothesis via historical evidence. Twin studies confirm high heritability rates for psychiatric disorders, providing estimations of the proportion of 'liability to a disorder' attributed to 'genetic effects' (see Table 2).

Table 2. Heritability estimates for selected psychiatric disorders

\begin{tabular}{lc}
\hline Disorder & $\begin{array}{c}\text { Heritability } \\
\text { estimate (\%) }\end{array}$ \\
\hline Schizophrenia & 80 \\
Bipolar disorder & 80 \\
Major depression & 40 \\
Generalized anxiety disorder & 30 \\
Panic disorder & 40 \\
Phobia & 35 \\
Alcohol problem or dependence & 60 \\
\hline
\end{tabular}

Heritability is the proportion of liability to a disorder accounted for by genetic effects. The heritability estimates are based on twin studies which employed DSM-III-R research diagnoses. NB: the heritabilities should be regarded only as approximations.

Source: Owen, Cardno \& O'Donovan (2000): 22, Table 1 
History is used to show that genetic factors are primary, moving environmental influences to the background, thus avoiding their potential to obscure the role of genetic transmission. As a demonstration of this formulation, consider the following extract:

In spite of these difficulties [that genetic complexity is compounded by nosological complexity], genetic epidemiological studies based upon operational research diagnoses have shown that genes play a role in many of the syndromes defined by psychiatric nosology. (Owen, Cardno and O'Donovan, 2000: 22)

The authors explain that common psychiatric disorders are complex, which is further 'compounded' by nosological complexities. The history of 'epidemiological studies' is introduced by contrast ('In spite of these difficulties') to moderate the implicit charge that complexity is an excuse for not identifying genes or that genes play no role in psychiatric disorders. Epidemiological research, of which twin studies are the gold-standard, are enrolled to reassert the genetic hypothesis ('have shown that genes play a role in many of the syndromes'), thereby defending genetic complexity while allowing environmental influences to play a mitigating role. The contrastive role of history is used in these 'moderate' formulations to defend complex gene interaction and reduce ambiguity.

In the 'assertive' formulation, similar assertions are made but the order is changed, as shown in the following sequence:

1. History shows that psychiatric disorders are heritable.

2. However, psychiatric disorders are complex.

3. Therefore, identifying genes for complex disorders is difficult.

Instead of defending genetic causation from past failures and present complexities, the history of twin and adoption studies is used to assert from the outset the robust nature of the genetic hypothesis. This provides a stronger footing for justifying current programmes of molecular research. Complexity is introduced to modify this assertion without casting doubt over the central assumption. Complexity is no longer framed as an excuse for a lack of progress, but warrants inevitable delays in geneidentification. The presence of 'the environment' as a possible causal factor occupies a mitigating role in the complexity of psychiatric disorders. This more assertive sequence of claims reflects the basic structural elements of this genre of scientific accounting. The following extract illustrates this formulation at work:

Twin and adoption studies consistently demonstrate a genetic influence on all major psychiatric disorders, confirming work that started in the 1930s. In fact, estimated heritability for bipolar disorder, schizo phrenia and autism ( $80 \%$ to $>90 \%)$ is much higher than that of breast cancer (5\% to $60 \%$ ) and Parkinson disease (13\% to $30 \%$ ), for which several genetic risk factors are now well established. In many respects, psychiatric disorders are similar to other complex traits that have been studied genetically: studies are complicated by locus heterogeneity, imprecisely specified traits, incomplete penetrance and interaction with non-genetic factors, resulting in a low contribution of each individual risk allele. (odds ratios <2) (Burmeister, M clnnis \& Zöllner, 2008: 527) 
The history of twin and adoption studies provides a solid foundation ('studies consistently demonstrate') applying to all major psychiatric disorders'. History is cast as uniform and continuous ('confirming work that started in the 1930s') to provide a strong basis for justifying contemporary research. Another technique frequently used in review articles is comparisons to medicine, which shore-up the resemblance between research into psychiatric disorders and more successful work carried out in medical genetics (e.g. 'breast cancer' and 'Parkinson disease'). The higher estimates of the heritability of psychiatric disorders are compared with the much lower estimates of heritability in the case of other medical disorders. This not only confirms genetic causation, but implies the future success of psychiatric genetics. The second part of the sequence introduces complexity via the modalized expression, 'In many respects', to assert the strong resemblance between psychiatric disorders and 'other complex traits' with a respectable pedigree of genetic research. The last part of the sequence elaborates the difficulties of studying complex traits ('studies are complicated by...') confirming the existence of multiple genes of small effect.

As we have shown, the history of twin and adoption studies is contrasted with accounts of complexity in two different ways, which are differentiated by moderate or assertive formulations. In the moderate version, history defends the genetic hypothesis from the criticism that genetic factors play no role, or that environmental factors play a stronger role, in the causation of psychiatric disorders. In the assertive version, history justifies the claim that psychiatric disorders have a strong genetic component, which, as is the case for some medical disorders for which genetic research has been more successful, are confounded by complex factors. Formulations of certainty and continuity reconstruct a version of history that sidesteps the criticisms that are often leveled at twin and adoption studies in order to establish a solid empirical foundation and to connect contemporary research with a strong, successful tradition. However, this version of history also accounts for the present: it reconfirms the central hypothesis that genes do play a role in psychiatric disorders; it reduces ambiguity surrounding explanations of complexity; and it allows complexity to explain the difficulties of disease prediction in terms of delay rather than failure.

\section{Prospective accounting}

Prospective accounting is another strategy within this genre of scientific narrative. Its main features are characterized by reprise (revisiting accounts of success and failure), condensation (summarizing the main arguments), and recommendation (identifying future areas of progress and caution). As we show, prospective accounting engages in the careful reconstruction of expectations in order to realign a narrative of progress and moderated optimism with the difficulties of complexity.

Within the field of psychiatric genetics, appealing to the future is shaped by the controversies of the past. The failure of the 'gene for' paradigm in the 80s and 90s has led to the emergence of more cautious forms of accounting. This responsibility is often performed by explicitly recognizing what the field has not achieved. This is the 
sobering description offered by one group of authors in Genetics in Medicine, a journal devoted to clinical applications of genetics:

\begin{abstract}
Although psychiatric genetics is characterized by unprecedented efforts to identify the underlying genetic basis, very few candidates have been accepted as definite risk genes. No genes that explain a major portion of the respective psychiatric disorder have emerged. Genes with a major effect might not exist; rather, increased risk for psychiatric conditions could result from a large number of small gene effects. The availability of new methods for genetic analysis on the genome level now allows for studies that were unimaginable a few years ago. It remains to be seen whether these new approaches will translate into real success. (Züchner et al., 2007: 338)
\end{abstract}

The authors contrast a sense of commitment and technological progress ('unprecedented efforts to identify') with the ineluctable failure to find 'definite risk genes'. Risk genes are presumed to be genes of major effect which, the authors claim, 'might not exist'. This is consistent with scientists' discourse on 'complexity', marking a provisional break with some of the troublesome aspects of M endelianism. But it also orients our attention to the uncertain future of mapping a 'large number of small gene effects'. Optimism is invested in the 'availability of new methods' for genome-wide scanning that will enable staggering scales of investigation that 'were unimaginable a few years ago'. In this 'update' to clinicians of what can be expected from the future, this passage explicitly avoids promise and adopts a more cautious 'wait and see' approach.

As much as responsibility entails the reluctance to make promises, it also involves highly publicized criticism of 'false' promises. This is clearly evident in the recent and ongoing controversial case of genetic testing. The following extract appears in one of the highest impact journals in genetic research, Nature Genetics, wherein a fully mobilized account of complexity warrants caution about the predictive capabilities of genetic tests:

Given the small individual effect sizes of the few identified risk variants and the complexities of overlapping genetic risk factors, phenotypes and environmental factors, it seems unlikely that genetic tests for diagnosing psychiatric disorders at an individual level will be informative any time soon - the launch of a test for a single unconfirmed rare variant seems premature. If large numbers of rare mutations are involved in the majority of cases, we might need to wait for cheap individual re-sequencing - the '\$1,000 genome', which is on the horizon. (Burmeister, M cInnis \& Zöllner, 2008: 537)

A fully developed description of complexity is contrasted with the simplicity of a Mendelian test for 'a single unconfirmed rare variant'. It is interesting to note that the kinds of criticism that have hampered the field more generally are now directed towards this untimely ('premature') development. The availability of a controversial commercial genetic test allows the authors to present themselves as responsible commentators on the field by implying that they adhere to more rigorous standards of what constitutes a 'confirmed' risk gene. Even if rare mutations are involved in psychiatric diagnoses, this more cautious account defers 'promising' until 'cheap individual re-sequencing' is available to scan the genome for 'a large number of rare mutations'. A common technique of prospective accounting is the deferral of promise until the advent of future technological developments, which are located 
perpetually 'on the horizon'. Earlier accounts of complexity were more radically sceptical about the possibilities for genetic testing:

Indeed, even when all susceptibility genes for a given disorder have been identified, it will still not be possible to predict the development of disease with certainty until the relevant environmental risk factors have also been identified and the nature of the various interactions understood. Such interactions may be as complex as chaotic systems like the weather, which is notoriously unpredictable over even the relatively short term. (Owen, Cardno \& O'Donovan, 2000: 29)

To the readers of M olecular Psychiatry, which positions itself at the interface of preclinical and clinical research, radical scepticism is maintained by contrasting the complicatedness of polygenic susceptibility with the non-linear complexity of disease prediction. The authors break from the reductionist model where prediction follows mechanistically from identifying 'all susceptibility genes'. Responsibility is manifest in the identification of the role of 'relevant environmental risk factors' and in the underscoring of the complexity of gene-environment interactions. The non-linear complexity of 'chaotic systems like the weather' introduces a qualitatively different description of multifactorial interactions to cast extreme doubt over disease prediction.

While some authors foreground complexity with moderate optimism, others consolidate the field's potential by foregrounding recent technological achievements. Systems biology, GWA studies, and the recent turn to 'endophenotypes', are all frequently cited as new directions by which promise is recast. The completion of the HGP, for instance, affords more efficient methods of gene-identification according to psychiatrists writing in the American Journal of M edical Genetics:

\begin{abstract}
Psychiatric genetics is coming to a period of great promise as the means to identify susceptibility genes is becoming easier. With the completion of the Human Genome Project, the knowledge of the HapM ap, and the imminent possibility of genome-wide association studies to complement genome-wide linkage studies, the ability to find the genes that cause psychiatric disorder becomes more and more likely ... With the acceptance that the DSM diagnoses may not be the most informative and that it is important to decompose those diagnoses into their component parts, once again the field is experiencing a resurgence of enthusiasm. The use of informative phenotypes that take into account measurement error and confounding has great potential to become the basis of a new strategy to find susceptibility genes for complex psychiatric disorders. We hope that as the field becomes more and more technical and statistical, it remembers that its roots are ultimately in clinical research. (Szatmari et al., 2007: 586)
\end{abstract}

Accounts of promise are less constrained when focusing on technological advances as opposed to the inherent difficulties of complexity. It is easier to speak of 'great promise' when the 'means to identify genes' represent a quantitative increase in the capacity to investigate more genes at any given time. The probability of finding susceptibility genes is invested in new molecular techniques of linkage and association ('knowledge of the HapMap, and the imminent possibility of genomewide association studies to complement genome-wide linkage studies'). But these technical achievements must also be informed by recent innovations at the clinical level. Prospective accounting seeks to establish a new alignment between genotype- 
phenotype by moving away from the largely subjective and non-quantitative criteria of the DSM. The authors achieve this move by asserting consensus in the field ('the acceptance that') and by reporting the apparent 'resurgence of enthusiasm' with which researchers have turned their attention to endophenotypes: biomarkers that lie closer to the genetic underpinnings of disease (Gottesman \& Gould, 2003). The 'great potential' of this 'new strategy' of gene-identification seeks to redefine the terrain of psychiatric nosology by establishing stable phenotypes with a clear genetic connection. However, the authors imply that as the field becomes increasingly reliant on bioinformaticians to interpret the huge volumes of data generated by genome-wide scans, there is a danger that the field may drift from its 'clinical' foundations. This warning comes at a time when psychiatric genetics is increasingly characterised as a multidisciplinary and, therefore, more complex, enterprise. While early descriptions of complexity can be seen as emerging justifications for large scale statistical (GWA) studies, there is a trade-off: as 'the ability to find the genes that cause psychiatric disorders becomes more and more likely', the taming of complexity will become an increasingly technical enterprise. The article, directed at both clinicians and geneticists, can be seen as maintaining clinical control by arguing that the future success of finding susceptibility genes relies on developing informative phenotypes.

Concerns about incorporating other specialties within the field often warrant appeals to integration. Expectations of progress are highlighted by mitigating organizational and disciplinary factors, such as disputes between geneticists and neuroscientists. Neuroscientists claim that genome-wide scans of large samples are untargeted, and are likely to uncover weakly associated genes that may have no relevance to functional biology. Geneticists, however, are not persuaded by the statistics on a few candidate genes whose functional links are poorly established; they opt instead for statistical power across much larger case-control studies (Wellcome Trust Case Control Consortium, 2007). Such disciplinary disputes over the choice between targeting a few genes of biological relevance or searching through many genes for statistical significance, allows authors to underscore the 'political' obstacles of progress. Appeals to integration in high profile journals, like Nature Genetics, attempt to rally the field by incorporating disciplines with expertise relevant to the search for the genetic aetiology of psychiatric disorders under the aegis of psychiatric genetics, by building large collaborations, and by establishing data-sets amenable to common analysis. In contrast to the clinicians who warn of psychiatric genetics drifting from its clinical foundations, the following extract challenges bioinformaticians to identify ways of integrating knowledge:

Currently, the fields of neuroscience, proteomics, gene expression analysis and genetics operate largely independently of each other. Once the functional pathways that are involved in psychiatric disorders and their associated traits of interest are identified, statistically sound combined analysis of genetics with gene expression and pathway analysis will be needed. Testing biologically plausible candidate genes for genetic association surely falls into this category, but this approach ... has led to many false positives and irreproducible reports, probably owing to a combination of genotyping error, publication bias and insufficient correction for multiple testing .... Merging different data types from separate fields into a common analysis that results in a joint 
statistical probability is a bioinformatic and statistical challenge (Burmeister et al., 2008:

537).

The lack of integration of psychiatric genetics is cast in terms of numerous fields that 'operate largely independently of each other'. Possible tensions arising from such divisions are glossed as technical and disciplinary. The authors accommodate the views of neuroscientists who advocate 'testing biologically plausible candidate genes for genetic association', but the form of responsible accounting we saw earlier suggests that attempts to do so have failed in the past ('this approach ... has led to many false positives and irreproducible reports'). The mention of such failures also warrants an explanation ('probably owing to a combination of genotyping error, publication bias and insufficient correction for multiple testing') upholding the geneticists' view that larger studies avoid false positives. The prospective strategy of overcoming the boundaries that separate these fields, notably the tensions between neuroscientists and geneticists, is a matter of 'triaging association studies' to incorporate 'analysis of genetics, with gene expression and pathway analysis'. Responsibility for taking up this 'challenge' is assigned to bioinformaticians, who are key to integrating the knowledge of these separate fields.

Appeals to integration also coincide with the logics of interdisciplinarity which are often framed in terms of accountability and innovation (Strathern, 2004; Barry et al., 2008). Interdisciplinarity promises a more accountable science, responsive to user needs while forming closer ties with economies of innovation. Breaking down the barriers of scientific specialization and thus promising the creation of greater interaction and unforeseen synergies, has clear rhetorical value. In the life sciences especially, where problems are constructed in terms of their complexity, the development of solution strategies often justify complex forms of social organization. This is implied by Zöllner, M clnnis and Burmeister (2008) who view the multiple levels of complexity as requiring equally complex strategies of investigation: 'It is unlikely that a single strategy will allow the identification of all genetic risk factors, but this complexity will have to be attacked from many different angles' (2008: 537). Thus, complexity in psychiatric genetics affords opportunities to produce novel assemblies of scientific specialties, capital and work.

\section{Discussion}

A feature of the articles examined is the consistent narrative that they present. The retrospective and prospective framings are important temporal formulations of an existential present: they reconstruct a history of disappointments and a future of hope. This narrative also has a further rhetorical dimension: it incorporates criticisms, while at the same time deleting or minimizing the controversies from which they are derived. This has the effect of producing a history of continuity and internal reflexivity, rather than a history of contingency and human error; past failures are recast as technical errors of a methodology informed by flawed assumptions about Mendelian inheritance; twin and adoption studies are routinely cited to provide evidence supporting the central genetic hypothesis; accounts of failure are converted into success as the field comes to recognise the underlying complexity of psychiatric disorders. 
In the narrative of simple-to-complex, complexity emerges as an explanation for the failure of linkage studies, later solidifying into a theoretical model of multifactorial and polygenic interaction. This web of genetic and non-genetic factors, the so called 'witches brew' (Owen, Cardno \& O'Donovan, 2000), constitutes a rescaling and reordering of the way in which psychiatric pathology is genetically constituted. It no longer makes sense to say that there is a 'gene for' a disorder. As Rose (2007: 204) has noted: 'the claim is now that mutations associated with increased susceptibility can be identified in precise loci in the base sequence of the genes that control the synthesis of the proteins involved in the production and transportation of neurotransmitters, receptors, enzymes, cell membranes or ion channels regulating the activity of neurons'. The emphasis on 'susceptibility' is indicative of a new gaze in which the molecularization of psychiatric pathology is continuously distributed, multiplied, and diffused.

As much as the post-HGP view of complexity constitutes a new way of seeing and speaking about psychiatric disorders, the focus of this paper is to show that 'complexity' performs rhetorical work in the way that it preserves hope for a research programme. This analysis demonstrates the ways in which a particular genre of scientific accounting accomplishes a number of strategic objectives:

- it exonerates the failure to identify genes for psychiatric disorders by suggesting a slowing down of progress as the science comes to grips with the sheer scale and distribution of susceptibilities throughout the human genome;

- it allows scientists to incorporate non-genetic factors, in theory, which insulates the field from criticisms of determinism by according these factors an indeterminate role;

- it presents susceptibility as being complex in nature, allowing scientists to engage in moderated forms of promising that explicitly avoid hype and thus appear cautious and responsible;

- it allows the field to attribute progress to processes that can address complexity via increased speed of production (e.g. cheaper and faster sequencing) and organizational reordering (e.g. multidisciplinary integration, large collaborations, and data sharing);

- it allows gene-identification programmes to continue under the hypothesis that multiple genes are implicated in disease susceptibility;

- it confers a kind of respectability to biological psychiatry by adopting the same rhetoric of complexity as that of the much larger and successful field of medical genetics.

The rise of complexity explanations within psychiatric genetics is a story of incorporation and neutralization of controversies. Complexity is cast as a sophisticated realization of the further work needed in the field; the simple-tocomplex trope extends this narrative, generating hope by directing attention to 'rapid advances' that will convert complexity into models of prediction and drug discovery.

However, the ambivalent descriptions of complexity found in many of the review articles not only imply uncertainty about the precise aetiology of psychiatric 
disorders, but also suggest ways of resolving tensions between different methodological viewpoints. The instability of the simple-complex boundary is sufficiently vague enough to incorporate Mendelian and polygenic models which, rather than generating tensions at an ontological level, may actually relieve tensions between competing research practices. In appealing to non-M endelian patterns of inheritance, complexity is neither a straightforward negation of Mendelianism nor a revolutionary 'paradigm shift', but a shift in focus. Hedgecoe (2001) has noted that multifactorial models allow single genes some role in the aetiology of schizophrenia. This is because 'enlightened' models of molecular analysis employ the same methods used to detect single genes of major effect.

The appeal of the 'essentialist gene model' (Kendler, 2006) describes much of the new generation of research, such as the high profile GWA studies. The problem with gene discovery programmes, Kendler argues, is that they assume a one-to-one relationship between genes and diagnostic categories; they are more committed to extending the 'parts list' of molecular biology than properly understanding the interactions among the parts. Essentialism reduces complexity via traditional methods of nomenclature and resemblance, assuming that disease categories are reliable entities. But pleiotropy (that single genes are implicated in multiple traits) suggests that the essentialist view does not capture the multidimensionality of gene function. Advances in molecular biology are undermining the simple definition of the gene. The complexity of 'gene sharing' would suggest that the boundaries and functions of genes are fuzzy and unpredictable. Kendler argues: 'Genes are not discrete entities like atoms of gold and silver. They are dynamic parts of biological systems of immense complexity. The discovery of specific genes that are involved in the etiology of psychopathology will not likely prove to be the basis on which to build an essentialist and categorical model of psychiatric diagnosis' (2006: 1144). These strong versions of complexity challenge the assumption that genes are natural kinds, and thus unproblematic units of investigation. More seriously, these accounts, which see complexity as something qualitatively different from the quantitative expansion of 'simplicity', are sceptical towards the foundation upon which the field builds its economies of hope. It is the prospects of anchoring psychiatric categories to a genecentred approach that drives the development of pharmaceutical interventions.

The appearance of genetic tests for neuropsychiatric disorders is an example of how different versions of complexity are more or less amenable to commercial exploitation. Genetic testing for neuropsychiatric disorders is a major goal for translational medicine, the so called push 'from bench to bedside'. The acceptance that common psychiatric disorders are complex suggested that it was unlikely that a genetic test would be offered to the public any time soon. Nonetheless, three companies - Psynomics, Neuromark. and Suregene - have recently begun offering genetic tests that explicitly target risk for major psychiatric disorders. These tests are expected to be the first wave of the commericalization of psychiatric genetic research. Until recently, genetic testing had received little attention within psychiatry, but the development of genome-wide scans, offered to the public via the internet by such companies as Navigenics, 23andMe, and deCODEme, and the launch of a test for bipolar disorder by Psynomics, has raised concerns about poor 
predictive value (Couzin, 2008; Braff \& Freedman, 2008; Burmeister et al., 2008). The dressing-up of what scientists now understand to be complex genetic susceptibility as something simple enough to be delivered by direct-to-consumer genetic tests shifts the burden of responsibility on to clinicians and patients to recognize this limited predictability. However, increasing 'complexity' by adding more genes is not an assurance of more accurate information, since pleiotropy and complex gene interactions increase the difficulty of interpretation. Braff and Freedman (2008) argue that, since the reduced complexity offers limited or spurious predictability, the 'simplicity' of the direct-to-consumer model may even be harmful to patients.

When scientists contribute to the historical narrative of psychiatric genetics they often foreground the complexity of the field as a means of constructing professional responsibility. Foregrounding complexity, especially in controversial areas of science, accepts responsibility for unknowns, displays flexibility towards present problems, and moderates future promises. A discourse of professional responsibility is rhetorically organized via elaborate sequences of 'hedging' or contrast structures i.e. juxtaposing areas of potential development with methodological uncertainty and limitation. These contrastive formulations produce the distinctive 'cautiously optimistic' framing of progress, often anticipating future problems while avoiding accusations of 'genohype'. It is worth asking to whom are these accounts of complexity directed? The range of publications indicates a transdisciplinary focus directed at scientists and clinicians across various disciplines: medicine, biology, genomics, and psychiatry. The fact that this genre of accounting is not found within the home journal Psychiatric Genetics is indicative of its outward focus. Contrasting formulations of complexity also perform different rhetorical functions. For instance, strong versions of complexity that foreground nonlinear causation emphasise 'extreme caution', while moderate versions of complexity that foreground 'complicatedness' emphasise 'moderate optimism'. Mainstream descriptions of 'complicatedness' are more inclined to justify exploring multiple gene models while retaining a focus on single gene approaches. Such descriptions are likely to appease opposing methodological camps and establish connections with wider professional communities.

If foregrounding complexity is a means of performing responsibility when engaging other professional communities, does complexity have this effect when engaging publics? Brian Wynne has argued that when scientists engage publics, complexity is routinely deleted, which 'effectively denies lack of predictive control, and thus also responsibility for it' (2005: 70). However, depending on the recipient, it can be a platform for authority, caution and competence, or an effacing admission of partial authority, uncertainty and confusion. Given the peculiar history of psychiatric genetics with its narrative of success, failure and moderated optimism, a reader is unlikely to infer a loss of scientific authority and control from the incorporation of complexity. An admission of complexity that serves as a reason for integrating different models, methods, and specialties can be seen as performing responsibility to a 'lurking public' which includes the interested readers of the scientific literature who are not psychiatric geneticists. In the case of the commercialization of genetic testing for psychiatric disorders, there are different stakes at risk on either side of 
the public/professional boundary. When commercial actors seek to profit from the results of molecular research the public are sold simple genetics, not the complexity so meticulously constructed in review articles. The way the professional community has responded to such recent developments illustrates the rhetorical power of complexity: they describe the complexity of psychiatric disorders in order to stress the poor predictive value of tests based on the simplicity of single 'genes for'. Genetic testing in its present form is presented as scientifically and clinically irresponsible, a 'bridge too far too soon' (Braff \& Freeman, 2008).

This paper demonstrates that scientific accounts of complexity are not merely descriptions of 'complicated' or 'complicating' factors in the aetiology of psychiatric disorders, they also perform professional responsibility. This illustrates an important aspect of the reflexivity of professionals as molecular biology grapples with the volume of information generated by high-throughput technologies in the postgenomic era. For some, it is tempting to pronounce a 'paradigm shift' to denote a move from simple to complex gene models. However, the discourse on complexity does not entail a straightforward shift from simple to complex. In addition to exonerating the field from past failures, complexity appears to offer a justification for combining different models, methods, and specialisms under the banner of 'psychiatric genetics'. Complexity is not simply a responsible admission of uncertainty, but a call for multidisciplinary integration: it seeks to articulate a common problem that requires a collaborative solution. The broad range of high impact journals in which this genre appears suggests a strategy of developing closer links between genetics, neuroscience, psychiatry, and molecular biology. Furthermore, as the field becomes increasingly inundated with data, it will rely on bioinformatics to resolve complexity. Our analysis suggests that complexity and reductionism are not mutually exclusive categories, but that they co-exist at the interstices and uncertainties of molecular biology. Descriptions of complexity are rhetorically organized justifications for marshalling new resources and reconstructing promises. We argue that this particular pattern of scientific accounting is not unique to professionals or the field of psychiatric genetics, but forms part of the everyday resources for managing uncertainty.

\section{Notes}

The support of the Economic and Social Research Council (ESRC) is gratefully acknowledged. The work was part of the Programme of the ESRC Research Centre for Economic and Social Aspects of Genomics. This work is also part of the Programme of the MRC Centre for Neuropsychiatric Genetics and Genomics. 


\section{References}

Aldous, Peter (1993) 'Disease Gene Search Goes Big Science', Science 259 (29 January): 591-92.

Alper, Joseph S \& Marvin R. Natowicz (1993) 'On Establishing the Genetic Basis of Mental Disease', Trends in Neuroscience 16(10): 387-89.

Baron, Miron, Nelson F. Freimer, Neil Risch, Bernard Lerer, Joyce R. Alexander, Richard E. Straub, Susha Asokan, Kamna Das, Amy Peterson, Jean Amos, Jean Endicott, Jurg Ott \& T. Conrad Gilliam (1993) 'Diminished Support for Linkage Between Manic Depressive IIIness and X-Chromosome Markers in Three Israeli Pedigrees', Nature Genetics 3: 49-55.

Baron, Miron, Neil Risch, Rahel Hamburger, Batsheva Mandel, Stuart Kushner, Michael Newman, Dov Drumer \& Robert H. Belmaker (1987) 'Genetic Linkage Between X-Chromosome Markers and Bipolar Affective Illness', Nature 326 (25 March): 289-92.

Barry, Andrew, Georgina Born \& Gisa Weszkalnys (2008) 'Logics of Interdisciplinarity', Economy and Society 37(1): 20-49.

Bazerman, Charles (1988) Shaping Written Knowledge: The Genre and Activity of the Experimental Article in Science (Madison WI: The University of Wisconsin Press).

Berkowitz, Ari (1996) 'Our Genes, Ourselves?', BioScience 46(1): 42-52.

Billig, Michael (1987) Arguing and Thinking: A Rhetorical Approach to Social Psychology (Cambridge: Cambridge University Press).

Billings, Paul R., Jonathan Beckwith \& Joseph S. Alper (1992) 'The Genetic Analysis of Human Behaviour: A New Era?', Social Science and Medicine 35(3): 227-38.

Blum, K., E.P. Noble, P.J. Sheridan, A. Montgomery, T. Ritchie, P. Jagadeeswaran, H. Nogami, A.H. Brigg, \& J.B. Cohn (1990) 'Allelic Association of Human Dopamine [D.sub.2] Receptor Gene in Alcoholism', Journal of the American Medical Association 263: 2055-60.

Boomsma, Dorret, Andreas Busjahn, Leena Peltonen (2002) 'Classical Twin Studies and Beyond', Nature Reviews Genetics 3(11): 872-82.

Braff, David L. \& Robert Freedman (2008) 'Clinically Responsible Genetic Testing in Neuropsychiatric Patients: A Bridge Too Far Too Soon', American Journal of Psychiatry, 165(8): 952-55.

Burmeister, Margrit, Melvin G. Mclnnis \& Sebastian Zöllner (2008) 'Psychiatric Genetics: Progress amid Controversy', Nature Reviews Genetics, 9: 527-40.

Cockcroft, Robert \& Susan M. Cockcroft (1992) Persuading People: An Introduction to Rhetoric (London: Macmillian).

Cook-Deegan, Robert M. (1994) The Gene Wars: Science, Politics and the Human Genome (New York, NY: W.W. Norton).

Couzin, Jennifer (2008) 'Gene Tests for Psychiatric Risk Polarize Researchers', Science, 319 (18 January): 274-77.

Cranor, Carl (1994) 'Genetic Causation', in C. Cranor (ed.), Are Genes Us? The Social Consequences of the New Genetics (New Brunswick, NJ: Rutgers University Press): $125-41$.

Detera-Wadleigh, Sevilla D., Wade H. Berrettini, Lynn R. Goldin, Denise Boorman, Stewart Anderson \& Elliot S. Gershon (1987) 'Close Linkage of c-Jarvey-ras-1 and the Insulin Gene to Affective Disorder is Ruled out in Three North American Pedigrees', Nature 325 (26 February): 806-08. 
Dick, Danielle M., Richard J. Rose \& Jaakko Kaprio (2006) 'The Next Challenge for Psychiatric Genetics: Characterizing the Risk Associated with Identified Genes', Annuals of Clinical Psychiatry 18(4): 223-31.

Dillon, George L. (1991) Contending Rhetorics: Writing in Academic Disciplines (Bloomington IN: Indiana University Press).

Egeland, Janice A, Daniela S. Gerhard, David L. Pauls, James N. Sussex, Kenneth K. Kidd, Cleona R. Alien, Abram M. Hostetter \& David E. Housman (1987) 'Bipolar Affective Disorder Linked to DNA Markers on Chromosome 11', Nature 325 (26 February): 783-87.

Evans, Kathryn L., David J. Porteous, Walter J. M uir, Douglas H. R. Blackwood (2001) 'Nuts and Bolts of Psychiatric Genetics: Building on the Human Genome Project', Trends in Genetics 17(1): 35-40.

Gelernter, Joel, David Goldman, Neil Risch (1993) 'The A1 Allele at the D2 Dopamine Receptor Gene and Alcoholism: A Reappraisal', Journal of the American M edical Association 269: 1673-77.

Gelernter, Joel, Stephanie O'Malley, Neil Risch, Henry R. Kranzler, John Krystal, Kathleen Merikangas, James L. Kennedy \& Kenneth K. Kidd (1991) 'No Association Between an Allele at the D2 Dopamine Receptor Gene (DRD2) and Alcoholism', Journal of the American M edical Association 266: 1801-07.

Gershon, Elliot S. \& Robert C. Cloninger (1994) Genetic Approaches to Mental Disorders (Washington, DC: American Psychiatric Press, Inc.).

Gilbert, G. Nigel \& Michael Mulkay (1984) Opening Pandora's Box: A Sociological Analysis of Scientists' Discourse (Cambridge: Cambridge University Press).

Gottesman, Irving I. \& Todd D. Gould (2003) 'The Endophenotype Concept in Psychiatry: Etymology and Strategic Intentions', American Journal of Psychiatry 160: 636-45.

Hedgecoe, Adam (2001) 'Schizophrenia and the Narrative of Enlightened Geneticization', Social Studies of Science 31(6): 875-911.

Hilgartner, Stephen (1990) 'The Dominant View of Popularization: Conceptual Problems, Political Uses', Social Studies of Science 20: 519-39.

Hodgkinson, Stephen, Robin Sherrington, Hugh Gurling, Roger Marchbanks, Stephen Reeders, Jacques Mallet, Melvin Mclnnis, Hannes Petursson, Jon Brynjolfsson (1987) 'Molecular Genetic Evidence for Heterogeneity in Manic Depression', Nature 325 (26 February): 805-06.

Joseph, Jay (2001) 'Separated Twins and the Genetics of Personality Differences: A Critique', American Journal of Psychology 114(1): 1-30.

Joseph, Jay (2003) The Gene Illusion: Genetic Research in Psychiatry and Psychology under the Microscope (Ross-on-Wye: PCCS Books).

Joseph, Jay (2006) The Missing Gene: Psychiatry, Heredity, and the Fruitless Search for Genes (New York, NY: Algora).

Kamin, Leon J. (1974) The Science and Politics of I.Q. (Potomac, MD: Earlbaum Associates).

Katz Rothman, Barbara (1995) 'Of M aps and Imaginations: Sociology Confronts the Genome', Social Problems 42(1): 1-10.

Kelsoe, John R, Edward I. Ginns, Janice A. Egeland, Daniela S. Gerhard, Alisa M. Goldstein, Sherri J. Bale, David L. Pauls, Robert T. Long, Kenneth K. Kidd, Giovanni Conte, et al. (1989) 'Re-evaluation of the Linkage Relationship Between 
Chromosome 11p loci and the Gene for Bipolar Affective Disorder in the Old Order Amish', Nature 342(16 November): 238-43.

Kendler, Kenneth S. (2006) 'Reflections on the Relationship Between Psychiatric Genetics and Psychiatric Nosology', American Journal of Psychiatry 163: 1138-46.

Kendler Kenneth S. \& Scott R. Diehl, (1993) 'The Genetics of Schizophrenia: A Current, Genetic-epidemiologic Perspective', Schizophrenia Bulletin 19(2): 26185.

Kennedy, James L., Luis A. Giuffra, Hans W. Moises, L. L. Cavalli-Sforza, Andrew J. Pakstis, Judith R. Kidd, Carmela M. Castiglione, Barbro Sjogren, Lennart Wetterberg, Kenneth K. Kidd (1988) 'Evidence Against Linkage of Schizophrenia to Markers on Chromosome 5 in a Northern Swedish Pedigree', Nature 336 (10 November): 167-70.

Kerr, Anne \& Tom Shakespeare (2002) Genetic Politics: From Eugenics to Genome (Cheltenham: New Clarion Press).

Kevles, Daniel J. (1995) In the Name of Eugenics: Genetics and the Uses of Human Heredity (Cambridge, MA: Harvard University Press).

Latour, Bruno \& Steve Woolgar (1986) Laboratory Life: The Construction of Scientific Facts (Princeton, NJ: Princeton University Press).

Lippman, Abby (1991) 'Prenatal Genetic Testing and Screening: Constructing Needs and Reinforcing Inequities', American Journal of Law and Medicine 17(1-2): 1550.

Myers, Greg (1991) 'Stories and Styles in Two M olecular Biology Review article', in C. Bazerman \& J. Paradis (eds), Textual Dynamics of the Professions: Historical and Contemporary Studies of $W$ riting in Professional Communities (Madison, WI: University of Wisconsin Press): 45-75.

Myers, Greg (1992) '“In this Paper we Report ...": Speech Acts and Scientific Acts', Journal of Pragmatics 17(4): 295-313.

O'Donovan, Michael C. \& Mike J. Owen (1999) 'Candidate-Gene Association Studies of Schizophrenia', American Journal of Human Genetics 65(3): 587-92.

Owen, Mike J., Alastair G. Cardno \& Mike C. O'Donovan (2000) 'Psychiatric Genetics: Back to the Future', M olecular Psychiatry 5: 22-31.

Plomin, Robert. (1990) 'The Role of Inheritance in Behavior', Science, 248 (13 April): 183-88.

Potter, Jonathan (1996) Representing Reality: Discourse, Rhetoric and Social Construction (London: Sage).

Ridley, M att (1996) The Origins of Virtue (London: Viking).

Ridley, M att (1994) The Red Queen (Harmondsworth: Penguin)

Robertson, Miranda (1987) 'Molecular Genetics of the Mind', Nature 325(26 February): 755.

Robertson, Miranda (1989) 'False Start on Manic Depression', Nature 342(16 November): 222.

Rose, Nicholas (2007) The Politics of Life Itself: Biomedicine, Power, and Subjectivity in the Twenty-First Century (Princeton, NJ: Princeton University Press).

Rose, Stephen (1995) 'The Rise of Neurogenetic Determinism', Nature 373(2 February): 380-82.

Rose, Stephen (1998) 'Neurogenetic Determinism and the New Euphenics', British Medical Journal 317: 1707-08. 
Rose, Stephen, Richard C. Lewontin \& Leon J. Kamin, (1984) Not In Our Genes: Biology, Ideology and Human Nature (London: Pantheon).

Sherrington, Robin, Jon Brynjolfsson, Hannes Petursson, Mark Potter, Keith Dudleston, Brian Barraclough, John Wasmuth, Mark Dobbs \& Hugh Gurling (1988) 'Localization of a Susceptibility Locus for Schizophrenia on Chromosome 5', Nature 336(10 November): 164-67.

Sinding, Christiane (1996) 'Literary Genres and the Construction of Knowledge in Biology: Semantic Shifts and Scientific Change', Social Studies of Science 26(1): 43-70.

Stoltenberg, Scott F. \& Margit Burmeister (2000) 'Recent Progress in Psychiatric Genetics - Some Hope but no Hype', Human M olecular Genetics 9(6): 927-35.

Strathern, Marilyn (2004) Commons and Borderlands: Working Papers on Interdisciplinarity, Accountability and the Flow of Knowledge (Wantage: Sean Kingston).

Szatmari, Peter, Michel Maziade, Lonnie Zwaigenbaum, Chantal Mérette, MarcAndre Roy, Ridha Joober \& Roberta Palmour (2007) 'Informative Phenotypes for Genetic Studies of Psychiatric Disorders', American Journal of Medical Genetics 144(5): 581-88.

Virgo, Julie A. (1971) 'The Review Article: Its Characteristics and Problems', The Library Quarterly 41(4): 275-91.

Wahlsten, Douglas (1979) 'A Critique of the Concepts of Heritability and Heredity in Behavioral Genetics', in J. R. Royce \& L. P. Mos. (eds), Theoretical Advances in Behavior Genetics (The Netherlands: Sijthoff \& Noordhoff): 425-70.

Wellcome Trust Case Control Consortium (2007) 'Genome-Wide Association Study of 14,000 Cases of Seven Common Diseases and 3,000 Shared Controls', Nature, 447 (7 June): 661-84.

Wilson Edward O. (1975) Sociobiology: The New Synthesis (Cambridge, M A: Harvard University Press).

Winerman, Lea (2004) 'A Second Look at Twin Studies', American Psychological Association M onitor 35(4): 46.

Wright, Robert (1996) The Moral Animal: Why We Are the Way We Are (London: Abacus).

Wynne, Brian (2005) 'Reflexing Complexity: Post-Genomic Knowledge and Reductionist Returns in Public Science', Theory, Culture and Society 22(5): 67-94.

Yoxen, Edward J. (1982) 'Giving Life a New Meaning: The Rise of the Molecular Biology Establishment', in N. Elias, H. Martins \& R. Whitley (eds), Scientific Establishments and Hierarchies (Dordrecht: Reidel): 123-43.

Züchner, Stephan, Sushma T. Roberts, Marcy C. Speer \& Jean C. Beckham (2007) 'Update on Psychiatric Genetics', Genetics in M edicine 9(6): 332-40. 